\title{
Surgery for herniated lumbar disc in private vs public hospitals: A pragmatic comparative effectiveness study
}

\author{
Mattis A. Madsbu ${ }^{1,2}$ (1) Øyvind Salvesen ${ }^{3} \cdot$ Sven M. Carlsen ${ }^{4,5} \cdot$ Steinar Westin $^{3} \cdot$ Kristian Onarheim $^{6}$. \\ Øystein P. Nygaard ${ }^{1,2,7} \cdot$ Tore K. Solberg $^{8,9,10}$. Sasha Gulati ${ }^{1,2,7}$
}

Received: 14 November 2019 / Accepted: 20 December 2019/Published online: 4 January 2020

(C) The Author(s) 2020

\begin{abstract}
Background There is limited evidence on the comparative performance of private and public healthcare. Our aim was to compare outcomes following surgery for lumbar disc herniation (LDH) in private versus public hospitals.

Methods Data were obtained from the Norwegian registry for spine surgery. Primary outcome was change in Oswestry disability index (ODI) 1 year after surgery. Secondary endpoints were quality of life (EuroQol EQ-5D), back and leg pain, complications, and duration of surgery and hospital stays.

Results Among 5221 patients, 1728 in the private group and 3493 in the public group, 3624 (69.4\%) completed 1-year followup. In the private group, mean improvement in ODI was 28.8 points vs 32.3 points in the public group (mean difference -3.5 , $95 \% \mathrm{CI}-5.0$ to $-1.9 ; P$ for equivalence $<0.001)$. Equivalence was confirmed in a propensity-matched cohort and following mixed linear model analyses. There were differences in mean change between the groups for EQ-5D (mean difference -0.05 , $95 \% \mathrm{CI}-0.08$ to $-0.02 ; P=0.002$ ) and back pain (mean difference $-0.2,95 \% \mathrm{CI}-0.2,-0.4$ to $-0.004 ; P=0.046$ ), but after propensity matching, the groups did not differ. No difference was found between the two groups for leg pain. Complication rates was lower in the private group $(4.5 \%$ vs $7.2 \% ; P<0.001)$, but after propensity matching, there was no difference. Patients operated in private clinics had shorter duration of surgery ( $48.4 \mathrm{vs} 61.8 \mathrm{~min})$ and hospital stay ( $0.7 \mathrm{vs} 2.2$ days).

Conclusion At 1 year, the effectiveness of surgery for LDH was equivalent in private and public hospitals.
\end{abstract}

Keywords Intervertebral disc displacement $\cdot$ Neurosurgery $\cdot$ Orthopedics $\cdot$ Public health $\cdot$ Sciatica

\section{Introduction}

Public health care is usually provided by the government through national healthcare systems, whereas private health care is often provided as "for profit" services. Ideological debates whether countries should strengthen public versus

This article is part of the Topical Collection on Spine degenerative

\author{
Mattis A. Madsbu \\ mattis.madsbu@gmail.com \\ Øyvind Salvesen \\ oyvind.salvesen@ntnu.no \\ Sven M. Carlsen \\ sven.carlsen@ntnu.no \\ Steinar Westin \\ steinar.westin@ntnu.no
}

private healthcare services are common. In times of economic recession with constraints on government budgets, disputes between the proponents of private and public health care systems tend to escalate. Discussions about resource allocation between private and public health providers should be evidence based, and focused on clinical effectiveness and costs.

\author{
Kristian Onarheim \\ kristian.onarheim@helse-midt.no \\ Øystein P. Nygaard \\ oystein.nygaard@ntnu.no \\ Tore K. Solberg \\ tore.solberg@unn.no \\ Sasha Gulati \\ sasha.gulati@ntnu.no
}

Extended author information available on the last page of the article 
There is currently limited and only poor-quality evidence regarding the comparative performance of the two health care systems [2]. In order to achieve a more informed policy, there is an urgent need for robust evidence by comparing the quality and effectiveness of the health care provided through both systems. Degenerative lumbar spine disorders are a leading cause of activity limitation and work absence throughout much of the world, and places an enormous economic burden on the whole society ranging from individuals, families, communities, industry, and all the way to governments. The most common reason for spine surgery is persisting or intolerable pain due to sciatica caused by lumbar disc herniation $[4,19]$. In many countries, surgical management of degenerative lumbar spine disorders is provided by both public and private hospitals, providing a unique opportunity to compare the two health care provider systems. The aim of this study was to compare patient-reported outcomes following surgery for lumbar disc herniation (LDH) because of sciatica in public versus private hospitals.

\section{Methods}

Reporting is consistent with the strengthening the reporting of observational studies in epidemiology (STROBE) statement. [25]

\section{Ethical approval}

This study was approved by the regional committee for medical research in central Norway (ID2016/840) and all participants provided written informed consent. The Data Inspectorate of Norway approved the registry protocol.

\section{Study population}

Norway has a public healthcare system with equal distribution of resources and uniform training and licensing of healthcare staff. The population is relatively homogenous and stable. Patients utilizing the public healthcare system are usually treated at the hospital serving their residential address, limiting referral bias. Surgery for LDH is provided free of cost to patients in the public healthcare system. In private hospitals, expenses of surgery (approximately USD 5400 for singlelevel lumbar microdiscectomy) are covered by the patients themselves or their insurance providers. Many of the patients treated at private hospitals have private health insurance paid for by their employers. During the study period, some of the private hospitals had government funding through contracts with the public regional health authorities.

We collected data through the Norwegian Registry for Spine Surgery (NORspine), a comprehensive registry for quality control and research [17]. In total, 37 of 40 centers performing lumbar spine surgery in Norway reported to NORspine during the study period. According to the Norwegian Directorate of Health, approximately $63 \%$ of all patients who underwent lumbar spine surgery in Norway during the study period were included in NORspine [17]. In general, the departments that participated in this study had the same preferred surgical strategy for LDH without radiological instability. Surgery was performed by ipsilateral paravertebral muscle retraction and removal of the disc herniation under microscope magnification by a unilateral transflaval approach [1]. Participation in the NORspine register was not mandatory for providers or patients, and it was not required for a patient to gain access to health care or for a provider to be eligible for payment. Follow-up time from the date of the operation was 1 year. Patients included in the study were treated at hospitals reporting at least 50 patients to NORspine during the study period.

We screened patients who underwent surgery between January 2007 and May 2014 for eligibility. Follow-up time from the date of the operation was 1 year.

We considered patients eligible for the study if they had a diagnosis of symptomatic paramedian lumbar disc herniation, surgery was performed as a single-level lumbar microdiscectomy, and their data were included in the NORspine registry. Patients were excluded who had undergone previous surgery of the lumbar spine, undergone fusion and/or open laminectomy as a surgical approach, or had associated spinal conditions (degenerative spondylolisthesis and/ or scoliosis).

\section{User involvement}

The Norwegian Back Pain Association (Ryggforeningen) reviewed the study protocol and provided feedback concerning study design and outcome measures.

\section{Outcome measures}

The primary outcome was change in disease-specific functional outcome between baseline and 12-month-follow-up measured with version 2.1 of the Oswestry disability index [5], which has been translated into Norwegian and tested for psychometric properties [8]. The Oswestry disability index questionnaire quantifies disability for degenerative conditions of the lumbar spine. It covers intensity of pain, ability to lift, ability to care for oneself, ability to walk, ability to sit, sexual function, ability to stand, social life, sleep quality, and ability to travel. For each topic, there are six statements describing potential scenarios, and patients select the one that most closely resembles their situation. The index is scored from 0 to 100 . Zero means no disability and 100 reflects maximum disability.

Secondary outcome measures were changes between baseline and 12-month follow-up in generic health-related quality 
of life, measured with the generic Euro-Qol-5D (EQ-5D), and intensity of back pain and leg pain. The Norwegian version of EQ-5D has shown good psychometric properties [20]. Intensity of pain was graded in two separate 0-10 numerical rating scales (NRS) for back pain and leg pain where 0 equals no pain and 10 represents the worst conceivable pain. The NRS pain scales and ODI have shown good validity and are frequently used in research on back pain $[8,20]$. We also compared duration of procedures, length of hospital stays, reoperation at the index level within 3 months of surgery, and surgical complication rates. Surgeons provided the following complications and adverse events to NORspine: intraoperative hemorrhage blood replacement or postoperative hematoma, unintentional durotomy, cardiovascular complications, respiratory complications, anaphylactic reactions, and wrong level for surgery. Patients reported the following complications if occurring within 3 months of surgery: wound infection, urinary tract infection, micturition problems, pneumonia, pulmonary embolism, and deep vein thrombosis.

\section{Data collection by NORspine}

On admission for surgery, the patients completed the baseline questionnaire, which included questions about demographics and lifestyle issues in addition to the outcome measures. During the hospital stay, using a standard registration form, the surgeon recorded data concerning diagnosis, previous lumbar spine surgery, comorbidity, American Society of Anesthesiologists (ASA) grade, image findings, and surgical approach and procedure. A questionnaire was distributed to patients by regular mail at 3 months and 1 year after surgery, completed at home by the patients, and returned in the same way. The patients who did not respond received one reminder with a new copy of the questionnaire. The patients completed baseline questionnaire data and postal follow-up questionnaires without any assistance from the surgeon or other staff from the treating hospital.

\section{Statistical analysis}

Statistical analyses were performed with SPSS version 23.0 (IBM Corporation, Chicago, IL, USA) and Software R [23]. The size of the study was based on a null hypothesis on nonequivalence and an alternative hypothesis of equivalence. If the population effect of treatment on changes in ODI was eight points or less, treatments were considered equivalent for effectiveness $[3,12,18]$. The sample size calculation relates to a two one-sided test for equivalence, with a significance level of $2.5 \%$. We computed the $P$ values for equivalence as 1 minus the maximum confidence level at which the confidence interval is contained in ( -8 to 8 ) divided by 2 giving the $P$ values of the two one-sided test for equivalence. This applied to both the complete case analysis and the mixed linear model analysis in both the aggregate cohort and the propensitymatched cohort. Assuming a correlation of 0.5 between baseline and follow-up measurements and a standard deviation of 18 for the individual measurements, this study has a $90 \%$ power, with 340 patients in each group.

For statistical comparison tests, we defined the significance level defined as $P \leq 0.05$ on the basis of a two-sided hypothesis test with no adjustments made for multiple comparisons. For the primary outcome and one secondary outcome (EQ5D), a statistician (ØS) blinded to treatment provider performed both a complete case analysis and a full information analysis using mixed linear models. Central tendencies are presented as means when normally distributed and as medians when skewed. We used the Chi square test for categorical variables. Baseline- and 1-year scores were compared with paired-samples $t$ test. Mean change scores between the groups were analyzed with independent-samples $t$ test for complete cases and mixed linear models on all available data. For mixed linear models, the combination of patients operated in private or public clinics and time was taken as fixed effect and participant ID specified as random effect. A multiple linear regression model was applied to assess the relationship between the change in ODI score at 1 year (dependent variable) and private or public treatment, controlling for potential confounders. The selection of predictors included was based on their clinical importance and association with the dependent variable $[6,10,16]$.

To achieve equality, we eliminate as many as possible confounding factors and provide best possible balance between the two groups; we generated propensity scores using logistic regression and adjusting for baseline covariates that could influence clinical outcomes, including age, sex, smoking, college education, partner, year of operation, BMI, ASA grade > 2 , relevant comorbidity, emergency operation, duration of sciatica $>1$ year, and preoperative ODI score. This was to achieve the closest approximate to a randomized clinical trial.

All covariates were entered into a logistic regression analysis, and we fitted a maximum likelihood model based on these covariates as predictors of private versus public treatment. The coefficients for these predictors of private versus public treatment was used to calculate a propensity score of 0 to 1 for each patient. Based on the calculated propensity scores, two evenly matched groups were formed for private and public treatment using a matching algorithm with the common caliper set at 0.010 . This dataset is referred to as the "propensity-matched cohort". We have analyzed continuous variables using a related sample two-tailed $t$ test for data with a normal distribution and continuous data exhibiting a skewed distribution using the Wilcoxon signed rank test for matched pairs. We used the McNemar's test for correlated proportions to compare discrete variables. We handled missing data with mixed linear models and did not perform multiple imputations. This strategy was in line with studies 
showing that it is not necessary to handle missing data using multiple imputations before performing a mixed model analysis on longitudinal data $[15,24]$.

\section{Results}

\section{Baseline characteristics}

A total of 5221 patients were included, 1728 operated in private hospitals and 3493 in public hospitals. Participants underwent surgery at 24 orthopedic or neurosurgical departments in 22 hospitals in Norway, 14 public and 8 private. Baseline characteristics were stratified by type of treatment center and matching (Table 1). In the aggregate cohort, there were significant differences between the two groups for baseline characteristics including age, sex, educational level, body mass index, tobacco use, comorbidity, American Society of Anesthesiologists grade, mean baseline Oswestry disability index score, mean baseline EQ-5D, mean NRS back and leg pain, and number of emergency surgery procedures. After propensity score matching (1281 pairs), these differences in baseline characteristics disappeared. The loss to follow-up rate in the aggregate cohort at 1 year was $30.9 \%(n=533)$ in the private group and $30.5 \%(n=1064)$ in the public group $(P=$ $0.77)$. In the propensity-matched cohort, the loss to follow-up rate in the private group was $32.5 \%(n=416)$ and $31.2 \%(n=$ $400)$ for the public group $(P=0.52)$. There were no differences between non-responders and responders at 1 year for preoperative ODI, preoperative back pain, preoperative leg pain, preoperative EQ-5D, comorbidity, or ASA grade.
However, there were differences between non-responders and responders in age (40.2 vs 45.6; $P<0.001)$, BMI $(27.0$ vs $26.5 ; P=0.05)$, female sex $(36.9 \%$ vs $42.5 \% ; P<0.001)$, college education $(33.8 \%$ vs $41.4 \% ; P<0.001)$, and tobacco smoking $(34.6 \%$ vs $26.4 \% ; P<0.001)$.

\section{Primary outcome}

Complete case analyses and mixed linear model analyses for outcomes in both the aggregate and propensity-matched cohorts at 1 year are presented in Table 2. Figures 1 and 2 show the primary outcomes in the aggregate and propensitymatched cohorts during 1 year of follow-up. For the private and public group combined, the mean improvement in ODI was 31.1 (95\% CI 30.4 to $31.9 ; P<0.001$ ) in the aggregate cohort and 28.4 (95\% CI 27.5 to $29.3 ; P<0.001)$ in the propensity-matched cohort. For the private group in the aggregate cohort, the improvement in ODI was 28.8 points vs 32.3 in the public group (mean difference $95 \% \mathrm{CI}-5.0$ to $1.9 ; P$ for equivalence $<0.001)$. Equivalence was confirmed in the propensity-matched cohort (mean difference $2.0,95 \%$ $\mathrm{CI}-0.25$ to $4.3 ; P<0.001$ for equivalence).

\section{Secondary outcomes}

There was a small difference in mean change between the groups in the aggregate cohort in favor of the public group for EQ-5D ( 0.25 vs 0.50 , mean difference $-0.05,95 \% \mathrm{CI}-$ 0.08 to $-0.02 ; P=0.002$ ) and back pain ( 3.3 vs 3.5 , mean difference $-0.2,95 \% \mathrm{CI}-0.22,-0.44$ to $-0.004 ; P=$ 0.046). After propensity matching, the groups did not differ

Table 1 Personal characteristics, coexisting illnesses, and measures of health status for both treatment groups in aggregate and propensity-matched cohorts. Values are numbers (percentages) unless stated otherwise

\begin{tabular}{|c|c|c|c|c|c|c|}
\hline \multirow[t]{2}{*}{ Variables } & \multicolumn{2}{|l|}{ Aggregate cohort } & \multirow[t]{2}{*}{$P$ value } & \multicolumn{2}{|c|}{ Propensity-matched cohort } & \multirow[t]{2}{*}{$P$ value } \\
\hline & $\begin{array}{l}\text { Private hospitals } \\
(n=1728)\end{array}$ & $\begin{array}{l}\text { Public hospitals } \\
(n=3493)\end{array}$ & & $\begin{array}{l}\text { Private hospitals } \\
(n=1281)\end{array}$ & $\begin{array}{l}\text { Public hospitals } \\
(n=1281)\end{array}$ & \\
\hline Age (years) & 43.3 & 44.3 & 0.01 & 42.9 & 42.9 & 0.96 \\
\hline Female sex & $622(36 \%)$ & $1506(4.1 \%)$ & $<0.001$ & $253(31.8 \%)$ & $255(32 \%)$ & 0.97 \\
\hline Life partner/married & $1313(76.5 \%)$ & $2605(75.3 \%)$ & 0.17 & $228(75.7 \%)$ & $225(75.5 \%)$ & 0.93 \\
\hline Attended college & $783(45.7 \%)$ & $1243(35.8 \%)$ & $<0.001$ & $200(26.8 \%) \mathrm{v}$ & $198(26.6 \%)$ & 0.96 \\
\hline Body mass index (BMI) & 26.3 & 26.7 & 0.001 & 26.4 & 26.4 & 0.84 \\
\hline Current smoker & $443(25.9 \%)$ & $1050(30.4 \%)$ & 0.001 & $228(24.2 \%)$ & $219(23.4 \%)$ & 0.71 \\
\hline Comorbidity & $247(14.3 \%)$ & $823(23.6 \%)$ & $<0.001$ & $134(12.5 \%)$ & $146(13.5 \%)$ & 0.51 \\
\hline ASA $>2$ & $29(1.7 \%)$ & $168(4.9 \%)$ & $<0.001$ & $21(1.7 \%)$ & $13(1.0 \%)$ & 0.23 \\
\hline Preoperative ODI & 40.9 & 47.5 & $<0.001$ & 41.3 & 41.4 & 0.91 \\
\hline Preoperative EQ-5D & 0.34 & 0.25 & $<0.001$ & 0.4 & 0.3 & 0.80 \\
\hline Preoperative back pain & 5.6 & 6.4 & $<0.001$ & 5.7 & 5.8 & 0.14 \\
\hline Preoperative leg pain & 6.5 & 7.0 & $<0.001$ & 6.5 & 6.5 & 0.60 \\
\hline Emergency surgery & $18(1.0 \%)$ & $1011(29.1 \%)$ & $<0.001$ & $11(0.8 \%)$ & $11(0.8 \%)$ & - \\
\hline
\end{tabular}




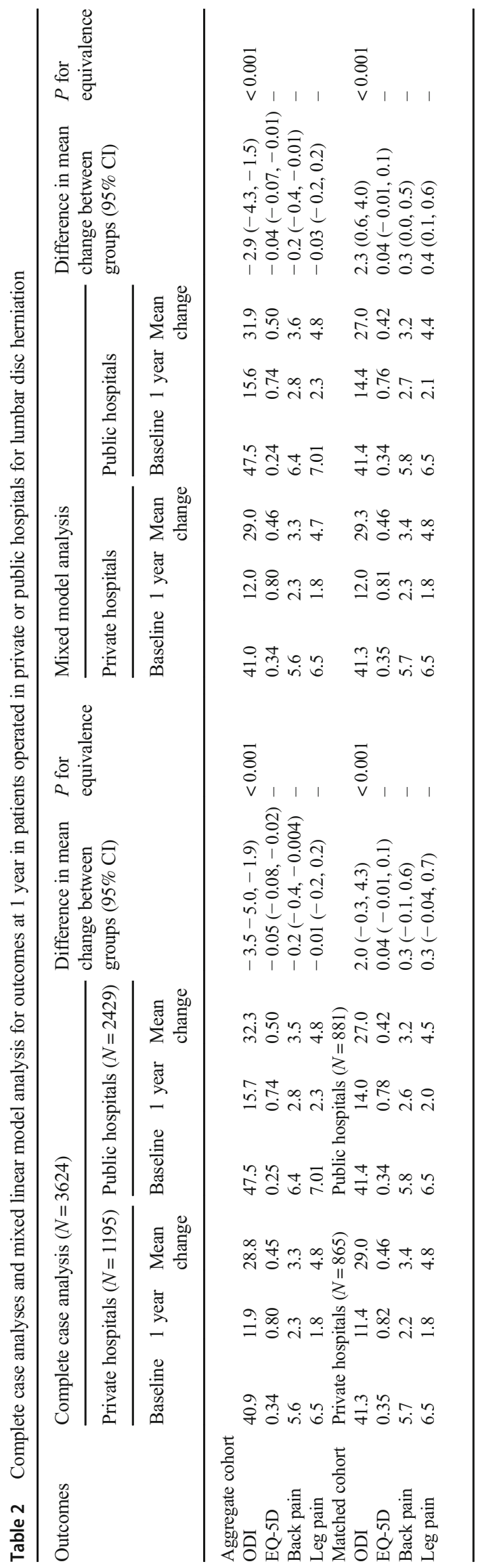

(0.46 vs 0.42 for EQ-5D; $P=0.13$ ) and (3.4 vs 3.2 for back pain; $P=0.16$ ). There was no difference between the two groups for leg pain. Duration of surgical procedures and length of hospital stays were lower in the private group compared to the public group (for the matched cohorts 47.9 vs $57.8 \mathrm{~min} ; P<0.001$ and 0.7 vs 2.0 days; $P<0.001$ ), as shown in Table 3. For the aggregate cohort, there was a significantly higher number of patients operated in a public hospital that experienced both perioperative complications and postoperative complications within 3 months ( $2.9 \%$ vs $1.4 \% ; P=0.001$ ) and $(6.5 \%$ vs $4.5 \% ; P=0.020)$, respectively. In the propensity-matched cohort, there were no differences in either perioperative or postoperative complications within 3 months between the two groups.

\section{Discussion}

To our knowledge, this is the first study to compare patientreported outcomes between private and public hospitals. Despite differences in patients' baseline characteristics that may influence treatment outcomes, the effectiveness of lumbar microdiscectomy was equivalent in public and private hospitals in this registry-based multicenter observational study. This finding was consistent in both unmatched and propensity-matched populations.

Duration of surgery was shorter in private hospitals. This may in part be explained by the surgical team's experience. Unlike private hospitals, most public hospitals are teaching institutions where surgical residents and operating room staff are learning the procedure and working under guidance and supervision. It is also possible that surgical units specializing in fewer procedures, microdiscectomy being one of them, are prone to develop a more efficient take on the surgical technique and logistics, resulting in shorter operation time. They also avoid the burden of having a readiness for acute interventions that in the public part of this cohort was as high as $29.1 \%$.

Longer hospital stays in public hospitals could partly be explained by the fact that public hospitals only received full reimbursement from Norwegian health authorities if the patients spent the first night following surgery in hospital. It is also rather common that patients originally referred to private hospitals are rejected when having comorbidities or other factors that may negatively influence outcomes and logistics [9]. Lumbar microdiscectomy seems to be a safe surgical procedure with few serious complications. In the aggregate cohort, there were slightly more perioperative complications within 3 months in the public group compared to the private group. These differences disappeared following propensity matching, supporting the evidence that "healthier" patients are treated in private hospitals [9]. 
Fig. 1 Change in Oswestry disability index score after microdiscectomy for lumbar disc herniation in aggregate cohort during 1-year follow-up for patients operated in private versus public hospitals

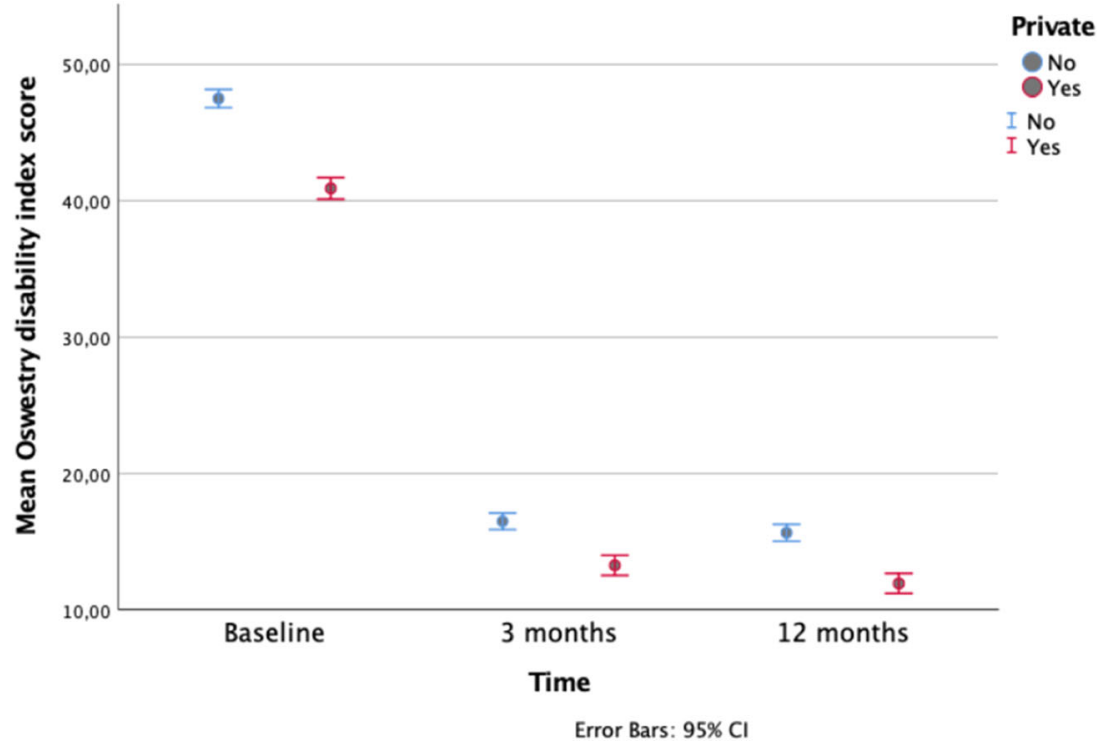

Unlike private hospitals, most public hospitals are teaching institutions where surgical residents and operating room staff are learning the procedure and working under guidance and supervision. Our results are in line with a study showing that surgical treatment of LDH at public hospitals with dedicated training programs does not lead to inferior patient care [22].

Economic and social differences between patients and access to healthcare are not as big of a challenge in Norway compared to other parts of the world. This could in part explain our equivalent results. However, a systematic review from low- and middle-income countries showed that the private sector was not superior to the public when considering medical efficiency [2].
Considering the equivalence of surgical results between the two health care providers in our nationwide patient sample, one could argue that there is a widespread access to high quality surgical management of LDH in both private and public health care systems. The dilemma each patient should consider is then not the effectiveness and quality of the health care, but rather their own economical capacity and possible waiting time for surgery. The role of private health insurance also comes under scrutiny in a country where the public health care system is well functioning and provides all emergency and complex in-house medical treatment [11].
Fig. 2 Change in Oswestry disability index score after microdiscectomy for lumbar disc herniation in propensity-matched cohort during 1-year follow-up for patients operated in private versus public hospitals

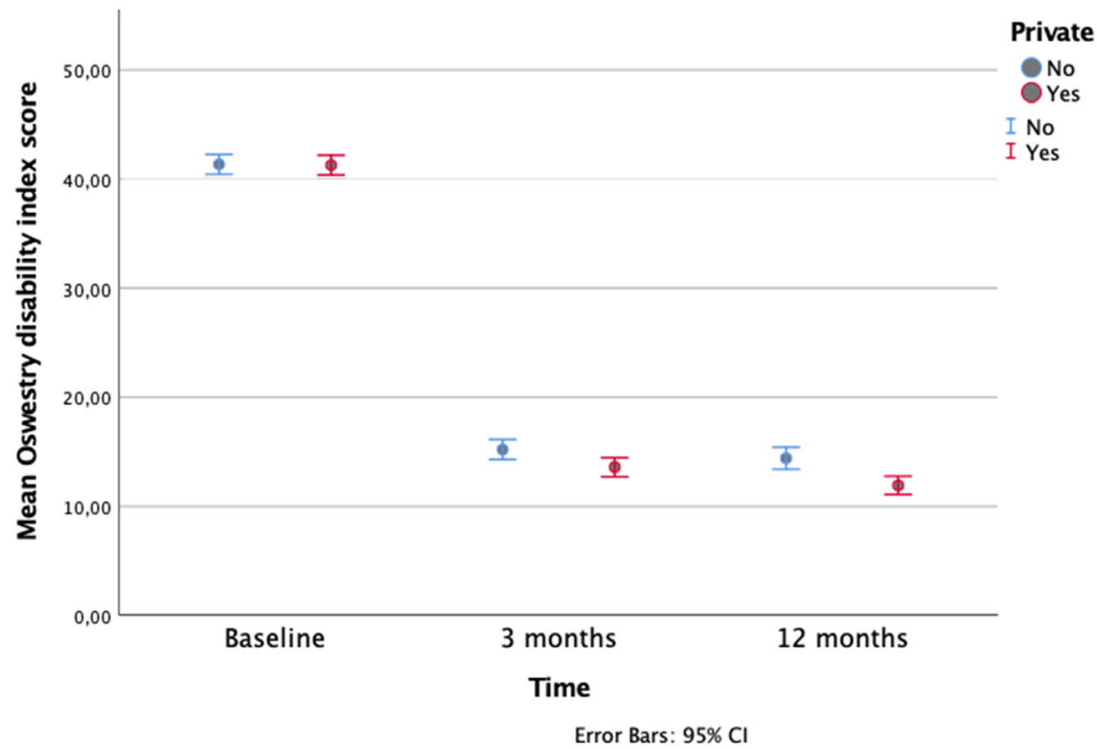


Table 3 Operation time, complications, and events. Values are numbers (percentages) of participants unless stated otherwise

\begin{tabular}{|c|c|c|c|c|c|c|}
\hline \multirow[t]{2}{*}{ Variables } & \multicolumn{2}{|l|}{ Aggregate cohort } & \multirow[t]{2}{*}{$P$ value } & \multicolumn{2}{|c|}{ Propensity-matched cohort } & \multirow[t]{2}{*}{$P$ value } \\
\hline & $\begin{array}{l}\text { Private hospitals } \\
(n=1728)\end{array}$ & $\begin{array}{l}\text { Public hospitals } \\
(n=3493)\end{array}$ & & $\begin{array}{l}\text { Private hospitals } \\
(n=1281)\end{array}$ & $\begin{array}{l}\text { Public hospitals } \\
(n=1281)\end{array}$ & \\
\hline Operation time (min) & 48.4 & 61.8 & $<0.001$ & 47.9 & 57.8 & $<0.001$ \\
\hline Days in hospital (no.) & 0.7 & 2.2 & $<0.001$ & 0.7 & 2.0 & $<0.001$ \\
\hline Patients with complications (no.) & $78(4.5 \%)$ & $250(7.2 \%)$ & $<0.001$ & $56(4.7 \%)$ & $76(6.2 \%)$ & 0.10 \\
\hline Perioperative complications (no.) & $24(1.4 \%)$ & $100(2.9 \%)$ & 0.001 & $18(1.4 \%)$ & $30(2.4 \%)$ & 0.11 \\
\hline Dural tear or CSF leak & $12(0.7 \%)$ & $61(1.7 \%)$ & 0.002 & $9(0.7 \%)$ & $21(1.7 \%)$ & 0.04 \\
\hline Nerve injury & $6(0.3 \%)$ & $7(0.2 \%)$ & 0.38 & $4(0.3 \%)$ & $2(0.2 \%)$ & 0.69 \\
\hline $\begin{array}{l}\text { Blood replacement or } \\
\text { postoperative hematoma }\end{array}$ & $8(0.5 \%)$ & $10(0.3 \%)$ & 0.32 & $6(0.5 \%)$ & $1(0.1 \%)$ & 0.13 \\
\hline Cardiovascular compl. & - & $5(0.1 \%)$ & 0.18 & - & - & - \\
\hline Respiratory compl. & $1(0.1 \%)$ & $1(-)$ & 0.55 & $1(0.1 \%)$ & - & - \\
\hline Anaphylactic reaction & $3(0.2 \%)$ & $3(0.1 \%)$ & 0.40 & $2(0.2 \%)$ & $1(0.1 \%)$ & 1.0 \\
\hline Wrong level surgery & - & $8(0.2 \%)$ & 0.06 & - & $1(0.1 \%)$ & - \\
\hline Complications within 3 months (no.) & $55(4.5 \%)$ & $157(6.5 \%)$ & 0.02 & $24(3.9 \%)$ & $33(5.3 \%)$ & 0.29 \\
\hline Wound infection & $41(3.4 \%)$ & $62(2.6 \%)$ & 0.17 & $18(2.9 \%)$ & $18(2.9 \%)$ & 1.0 \\
\hline Urinaty tract infection & $3(0.2 \%)$ & $47(1.9 \%)$ & $<0.001$ & $2(0.3 \%)$ & $8(1.2 \%)$ & 0.11 \\
\hline Micturition problems & $10(0.8 \%)$ & $47(1.9 \%)$ & 0.01 & $4(0.6 \%)$ & $7(1.1 \%)$ & 0.55 \\
\hline Pneumonia & $3(0.2 \%)$ & $10(0.4 \%)$ & 0.56 & $2(0.3 \%)$ & $4(0.6 \%)$ & 0.69 \\
\hline Pulmonary embolism & $1(0.1 \%)$ & $0(-)$ & 0.33 & - & - & - \\
\hline Deep venous thrombosis & - & $2(0.1 \%)$ & 1.0 & - & - & - \\
\hline Reoperated within 90 days no. (\%) & $20(1.2 \%)$ & $44(1.3 \%)$ & 0.79 & $10(0.8 \%)$ & $10(0.8 \%)$ & 1.0 \\
\hline
\end{tabular}

\section{Study strengths and limitations}

The major strength of this study is our use of propensity-matched groups to minimize confounding factors. Other strengths include the large sample size, pragmatic study design based on prospective registry data with high external validity, use of patient-reported outcome measures, and protocol-based statistical analyses with blinded assessment of main outcome measures. The main limitation was the lack of randomization. Even though propensity-matched patient groups adjusts for known interactions, while unlikely, residual or introduction of confounding cannot be ruled out. Another weakness was the loss to follow-up of $30.6 \%$ of participants regarding Oswestry disability index scores at 1 year. A previous study on a similar population from the NORspine registry showed no difference in outcomes between non-responders and responders [21]. The minor differences in baseline characteristics between non-responders and responders at 1 year are not likely to influence our results [7, 13-15]. Also, we are lacking data on exact amounts of costs, payment, and reimbursements, inhibiting us from performing cost-effectiveness analyses.

\section{Conclusion}

At 1 year, the effectiveness of microdiscectomy for lumbar disc herniation was equivalent for patients operated in private compared to public hospitals. However, patients operated in private clinics were managed more efficiently. Favorable outcomes were observed at 1 year in both treatment groups.

Acknowledgments We thank the Norwegian Registry for Spine Surgery (NORspine). We thank all patients and spine surgeons who participate in NORspine registration.

Data sharing All data is shared.

Authors' contribution All authors read and approved the final manuscript. MAM is the guarantor and originally conceived the study with SG. SG and MAM were involved in the study design, collection of data, statistical analysis, and writing of the manuscript. TKS and ØPN took part in collection of data and writing of manuscript. ØS and SMC took part of study design, statistical analyses and writing of manuscript. SW and KO were involved with study design and writing of manuscript.

Funding information The registry receives funding from the University of Northern Norway and Norwegian health authorities. 


\section{Compliance with ethical standards}

Conflict of interest The authors declare that they have no conflict of interest.

Ethical approval This study was approved by the regional committee for medical research in central Norway (ID2016/840) and all participants provided written informed consent. The Data Inspectorate of Norway approved the registry protocol.

Open Access This article is licensed under a Creative Commons Attribution 4.0 International License, which permits use, sharing, adaptation, distribution and reproduction in any medium or format, as long as you give appropriate credit to the original author(s) and the source, provide a link to the Creative Commons licence, and indicate if changes were made. The images or other third party material in this article are included in the article's Creative Commons licence, unless indicated otherwise in a credit line to the material. If material is not included in the article's Creative Commons licence and your intended use is not permitted by statutory regulation or exceeds the permitted use, you will need to obtain permission directly from the copyright holder. To view a copy of this licence, visit http://creativecommons.org/licenses/by/4.0/.

\section{References}

1. Arts MP, Brand R, van den Akker ME, Koes BW, Bartels RH, Peul WC, Leiden-The Hague Spine Intervention Prognostic Study G (2009) Tubular diskectomy vs conventional microdiskectomy for sciatica: a randomized controlled trial. JAMA 302:149-158. https:// doi.org/10.1001/jama.2009.972

2. Basu S, Andrews J, Kishore S, Panjabi R, Stuckler D (2012) Comparative performance of private and public healthcare systems in low- and middle-income countries: a systematic review. PLoS Med 9:e1001244. https://doi.org/10.1371/journal.pmed.1001244

3. Brox JI, Reikeras O, Nygaard O, Sorensen R, Indahl A, Holm I, Keller A, Ingebrigtsen T, Grundnes O, Lange JE, Friis A (2006) Lumbar instrumented fusion compared with cognitive intervention and exercises in patients with chronic back pain after previous surgery for disc herniation: a prospective randomized controlled study. Pain 122:145-155. https://doi.org/10.1016/j.pain.2006.01.027

4. Deyo RA, Mirza SK (2016) Clinical practice. Herniated lumbar intervertebral disk. N Engl J Med 374:1763-1772. https://doi.org/ 10.1056/NEJMcp1512658

5. Fairbank JC, Couper J, Davies JB, O'Brien JP (1980) The Oswestry low back pain disability questionnaire. Physiotherapy 66:271-273

6. Giannadakis C, Nerland US, Solheim O, Jakola AS, Gulati M, Weber C, Nygaard OP, Solberg TK, Gulati S (2015) Does obesity affect outcomes after decompressive surgery for lumbar spinal stenosis? A multicenter, observational, registry-based study. World Neurosurg 84:1227-1234. https://doi.org/10.1016/j.wneu.2015.06. 020

7. Giannadakis C, Solheim O, Jakola AS, Nordseth T, Gulati AM, Nerland US, Nygaard OP, Solberg TK, Gulati S (2016) Surgery for lumbar spinal stenosis in individuals aged 80 and older: a multicenter observational study. J Am Geriatr Soc 64:2011-2018. https://doi.org/10.1111/jgs.14311

8. Grotle M, Brox JI, Vollestad NK (2003) Cross-cultural adaptation of the Norwegian versions of the Roland-Morris disability questionnaire and the Oswestry disability index. J Rehabil Med 35: 241-247

9. Grotle M, Solberg T, Storheim K, Laerum E, Zwart JA (2014) Public and private health service in Norway: a comparison of patient characteristics and surgery criteria for patients with nerve root affections due to disc herniation. Eur Spine J 23:1984-1991. https://doi.org/10.1007/s00586-014-3293-z

10. Gulati S, Madsbu MA, Solberg TK, Sorlie A, Giannadakis C, Skram MK, Nygaard OP, Jakola AS (2017) Lumbar microdiscectomy for sciatica in adolescents: a multicentre observational registry-based study. Acta Neurochir 159:509-516. https:// doi.org/10.1007/s00701-017-3077-4

11. Gulati S, Solheim O, Carlsen SM, Oie LR, Jensberg H, Gulati AM, Madsbu MA, Giannadakis C, Jakola AS, Salvesen O (2018) Risk of intracranial hemorrhage (RICH) in users of oral antithrombotic drugs: nationwide pharmacoepidemiological study. PLoS One 13: e0202575. https://doi.org/10.1371/journal.pone.0202575

12. Knutsson B, Michaelsson K, Sanden B (2013) Obesity is associated with inferior results after surgery for lumbar spinal stenosis: a study of 2633 patients from the Swedish spine register. Spine (Phila Pa 1976) 38:435-441. https://doi.org/10.1097/BRS. 0b013e318270b243

13. Madsbu MA, Oie LR, Salvesen O, Vangen-Lonne V, Nygaard OP, Solberg TK, Gulati S (2018) Lumbar microdiscectomy in obese patients: a multicenter observational study. World Neurosurg 110: e1004-e1010. https://doi.org/10.1016/j.wneu.2017.11.156

14. Madsbu MA, Salvesen O, Werner DAT, Franssen E, Weber C, Nygaard OP, Solberg TK, Gulati S (2018) Surgery for herniated lumbar disc in daily tobacco smokers: a multicenter observational study. World Neurosurg 109:e581-e587. https://doi.org/10.1016/j. wneu.2017.10.024

15. Madsbu MA, Solberg TK, Salvesen O, Nygaard OP, Gulati S (2017) Surgery for herniated lumbar disk in individuals 65 years of age or older: a multicenter observational study. JAMA Surg 152: 503-506. https://doi.org/10.1001/jamasurg.2016.5557

16. Nerland US, Jakola AS, Giannadakis C, Solheim O, Weber C, Nygaard OP, Solberg TK, Gulati S (2015) The risk of getting worse: predictors of deterioration after decompressive surgery for lumbar spinal stenosis: a multicenter observational study. World neurosurgery 84:1095-1102. https://doi.org/10.1016/j.wneu.2015.05.055

17. Nerland US, Jakola AS, Solheim O, Weber C, Rao V, Lonne G, Solberg TK, Salvesen O, Carlsen SM, Nygaard OP, Gulati S (2015) Minimally invasive decompression versus open laminectomy for central stenosis of the lumbar spine: pragmatic comparative effectiveness study. BMJ 350:h1603. https://doi.org/10.1136/bmj.h1603

18. Ostelo RW, Deyo RA, Stratford P, Waddell G, Croft P, Von Korff M, Bouter LM, de Vet HC (2008) Interpreting change scores for pain and functional status in low back pain: towards international consensus regarding minimal important change. Spine (Phila Pa 1976) 33:90-94. https://doi.org/10. 1097/BRS.0b013e31815e3a10

19. Peul WC, van Houwelingen HC, van den Hout WB, Brand R, Eekhof JA, Tans JT, Thomeer RT, Koes BW, Leiden-The Hague Spine Intervention Prognostic Study G (2007) Surgery versus prolonged conservative treatment for sciatica. N Engl J Med 356: 2245-2256. https://doi.org/10.1056/NEJMoa064039

20. Solberg TK, Olsen JA, Ingebrigtsen T, Hofoss D, Nygaard OP (2005) Health-related quality of life assessment by the EuroQol5D can provide cost-utility data in the field of low-back surgery. Eur Spine J 14:1000-1007. https://doi.org/10.1007/s00586-0050898-2

21. Solberg TK, Sorlie A, Sjaavik K, Nygaard OP, Ingebrigtsen T (2011) Would loss to follow-up bias the outcome evaluation of patients operated for degenerative disorders of the lumbar spine? Acta Orthop 82:56-63. https://doi.org/10.3109/17453674.2010. 548024

22. Stienen MN, Smoll NR, Hildebrandt G, Schaller K, Gautschi OP (2014) Early surgical education of residents is safe for microscopic lumbar disc surgery. Acta Neurochir 156:1205-1214. https://doi. org/10.1007/s00701-014-2070-4 
23. Team. RDC (2007) R a language and environment for statistical computing. R Foundation for Statistical Computing

24. Twisk J, de Boer M, de Vente W, Heymans M (2013) Multiple imputation of missing values was not necessary before performing a longitudinal mixed-model analysis. J Clin Epidemiol 66:1022-1028. https://doi.org/10.1016/j. jclinepi.2013.03.017

25. von Elm E, Altman DG, Egger M, Pocock SJ, Gotzsche PC, Vandenbroucke JP, Initiative S (2007) The strengthening the reporting of observational studies in epidemiology (STROBE) statement: guidelines for reporting observational studies. Lancet 370:1453-1457. https://doi.org/10.1016/S0140-6736(07)61602-X

\section{Comments}

The microsurgical treatment of lumbar disc herniation (LDH) is one of the most commonly performed procedures in neurosurgery. For its relatively minor degree of complexity, lumbar microdiscectomy can be performed by supervised trainees without increasing the risk of complications and adverse outcome. Training the next generation of spine surgeons typically takes place in public hospitals and - as this paper illustrates - on a selected cohort of patients that are older, smoke more, have a lower educational level but suffer from more comorbidities and higher baseline case severity. Considering the teaching aspect, it is not surprising that the OR time is longer (62 vs. $48 \mathrm{~min}$ ) \& the rate of incidental durotomies is somewhat higher in patients treated at public hospitals ( $1.7 \%$ vs. $0.7 \%)$, even in the propensity-matched models. Nonetheless, both - patients treated in public or private hospitals - benefit substantially from the operation and the rates of nerve injuries (ca. $0.2 \%$ ) or 90-day re-operations (ca. 1.2\%) are similarly low. To me, this work underlines today's high standards of surgical teaching in the OR, which becomes increasingly important in times of decreasing working time \& OR exposure of neurosurgical trainees.

Martin N. Stienen

Zurich / Stanford

Publisher's note Springer Nature remains neutral with regard to jurisdictional claims in published maps and institutional affiliations.

\section{Affiliations}

\section{Mattis A. Madsbu ${ }^{1,2}$ (D) $\bullet$ yvind Salvesen ${ }^{3} \cdot$ Sven M. Carlsen ${ }^{4,5} \cdot$ Steinar Westin $^{3} \cdot$ Kristian Onarheim $^{6}$. $\emptyset_{\text {ystein P. Nygaard }}{ }^{1,2,7} \cdot$ Tore K. Solberg $^{8,9,10} \cdot$ Sasha Gulati ${ }^{1,2,7}$}

1 Department of Neurosurgery, St. Olavs University Hospital, Trondheim, Norway Department of Neuroscience, Norwegian University of Science and Technology (NTNU), Trondheim, Norway

3 Department of Public Health and Nursing, Norwegian University of Science and Technology (NTNU), Trondheim, Norway

4 Department of Clinical and Molecular Medicine, Faculty of Medicine and Health Sciences, Norwegian University of Science and Technology (NTNU), Trondheim, Norway

5 Department of Endocrinology, St Olavs Hospital, Trondheim, Norway
6 Central Norway Regional Health Authority, Stjørda, Norway

7 National Advisory Unit on Spinal Surgery, St. Olavs University Hospital, Trondheim, Norway

8 The Norwegian National Registry for Spine Surgery, University Hospital of Northern Norway (UNN), Tromsø, Norway

9 Department of Neurosurgery, University Hospital of Northern Norway (UNN), Tromsø, Norway

10 Institute of Clinical Medicine, The Arctic University of Norway (UIT), Tromsø, Norway 